\title{
Correspondence
}

\section{Heparin reversal protocol}

To the Editor:

The number of ways of manipulating the coagulation system during and after cardiopulmonary bypass must approximate the number of cardiac anaesthetists. However, Bull et al.' showed that protocols for heparin administration and reversal based on patient age, height, weight or BSA were inadequate. They suggested the use of a heparin dose-response plot created by measuring activated clotting time (ACT) to maintain safe anticoagulation and to predict the dose of protamine required for heparin reversal. ${ }^{2}$ This requires the construction of a graph. I offer the following formulac based on their technique.

Consider the Figure, where $x$ represents an ACT result and $y$ the corresponding dose of heparin.

Let

$A=\left(x_{0}, 0\right)$; the control $A C T$, no heparin

$\mathrm{B}=\left(\mathrm{x}_{1}, \mathrm{y}_{1}\right)$; following the first heparin dose

$\mathrm{C}=\left(\mathrm{x}_{2}, \mathrm{y}_{2}\right)$; following a second heparin dose

$D=\left(x_{d}, y_{d}\right)$; representing the last ACT prior to reversal, and the remaining heparin

Bull averages $\overline{\mathrm{AB}}$ and $\overline{\mathrm{AC}}$ by bisecting $\angle \mathrm{BAC}$ to produce $\overline{\mathrm{AD}}$, which he calls the heparin dose-response line. What Bull does with his graph can be accomplished with simple algebra.

First, say that you know $A$ and $B$ and want to arrive at a

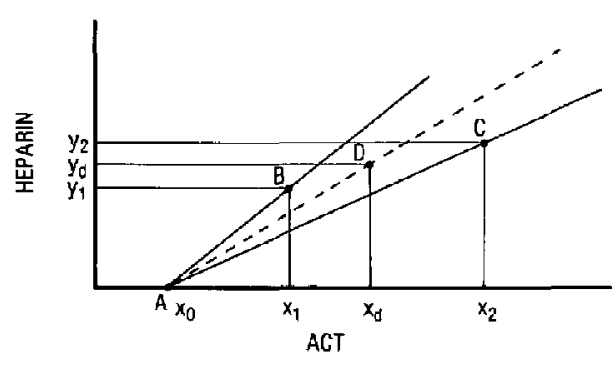

FIGURE Relationship of ACT result to heparin requirement. Sec Icx for explanation. point $\left(x_{n}, y_{n}\right)$ where $x_{n}=480$ seconds and $x_{1}<480$ seconds. Since $\left(x_{0}, 0\right),\left(x_{1}, y_{1}\right)$ and $\left(x_{n}, y_{n}\right)$ are colinear, then

$\frac{y_{n}-0}{x_{n}-x_{0}}=\frac{y_{1}-0}{x_{1}-x_{0}}$

and

$y_{n}=y_{1} \cdot\left(\frac{x_{n}-x_{0}}{x_{1}-x_{0}}\right)$

Using this formula, any desired ACT can be achieved. The additional heparin required is $y_{n}-y_{1}$.

Now, at the end of bypass, when you want to reverse the heparin (assuming the patient is normothermie), you need to determine $D\left(x_{d}, y_{d}\right)$, where $x_{d}$ is the last $A C T$. Since $\overline{\mathrm{AD}}$ bisects $\angle \mathrm{BAC}$, then the slope of $\overline{\mathrm{AD}}$,

$\mathrm{m}_{\overline{\mathrm{AD}}}=\frac{\mathrm{m}_{\overline{\mathrm{AB}}}+\mathrm{m}_{\overline{\mathrm{AC}}}}{2}$.

Since

$\mathrm{m}=\frac{\Delta \mathrm{y}}{\Delta \mathrm{x}}$

then

$\frac{y_{d}-0}{x_{d}-x_{0}}=\frac{\frac{y_{1}-0}{x_{1}-x_{0}}+\frac{y_{2}-0}{x_{2}-x_{0}}}{2}$

This becomes

$y_{d}=\frac{x_{d}-x_{0}}{n}\left(\frac{y_{1}}{x_{1}-x_{0}}+\frac{y_{2}}{x_{2}-x_{0}}\right)$

$\mathrm{y}_{\mathrm{d}}$ is the amount of heparin remaining in the circulation. If all $y$ values are in units of $1000 \mathrm{lU}$, then simply multiply $y_{d}$ by the protamine neutralizing factor (PNF) to determine the dose of protamine required.

If one feels that averaging more of Bull's lines will produce a more accurate dose-response line, the formula can easily be adapted:

$y_{d}=\frac{x_{d}-x_{0}}{n}\left(\frac{y_{1}}{x_{1}-x_{0}}+\frac{y_{2}}{x_{2}-x_{0}}+\cdots+\frac{y_{n}}{x_{n}-x_{0}}\right)$

These two formulae can easily be permanently programmed into a handheld computer so that all the anaesthetist need do is type in the $x$ and $y$ values. This circumvents the need to draw out a graph for each case. 
David S. Pounder MD

Department of Anaesthesia

Victoria General Hospital and

Dalhousie University

Halifax, Nova Scotia

B3H 2 Y9

\section{REFERENCES}

1 Bull BS, Korpman RA, Huse WM, Briggs BD. Heparin therapy during extracorporeal circulatiun I. Problems inherent in existing heparin protocols. J Thorac Cardiovasc Surg 1975; 5: 674-84.

2 Butl BS, Huse WM, Brauer FS, Korpman RA. Heparin therapy during extracorporeal circulation II. The use of a dose-response curve to individualize heparin and protamine dosage. J Thorac Cardiovase Surg 1975; 5: 685-9.

\section{Prolonged bleeding from epidural insertion site}

To the Editor:

We wish to report the interesting case report of a patient who developed prolonged bleeding from the skin puncture site during continuous epidural anaesthesia for labour.

The patient is a 35-year-old Gravida II, Para I, who presented in labour with vital signs of BP 140/90, HR 80, temperature $36^{\circ} \mathrm{C}$, urine analysis negative. She was in excellent health and was taking no medications. She requested an epidural for pain relief and a 16-gauge Tuohy epidural needle was inserted at the $\mathrm{L}_{4-5}$ interspace after local infiltration and a skin puncture made with a 17-gauge needle. The epidural needle was inserted without difficulty and the catheter was placed without incident. Twelve $\mathrm{ml}$ of 0.25 per cent bupivacaine was injected in two doses of $3+9 \mathrm{ml}$. The epidural catheter was fixed with Opsite ${ }^{\text {ti }}$ and adhesive tape and the patient was comfortable. Prior to the next top-up dose (about one hour), the aurse noted that the patient was "soaked" in blood, the source of which appeared to be the epidural site. The estimated blood loss from this cause was about $200 \mathrm{ml}$. When the Opsite was removed and the skin examined, to our surprise, there was a small artery bleeding, and bright red blood was spurting from the skin puncture site. Pressure was applied for $\frac{1}{2}$ hour without effect, so we removed the catheter and placed a pursestring suture around the puncture site. A second epidural was established at $\mathrm{L}_{3-4}$, uneventfully. No further blecding occurred and no complications arose as a result of the incident. The patient subsequently underwent a Caesarean section. The suture was removed 48 hours later and no further bleeding was seen. Perhaps an alternative could have been to leave in the first catheter and put a suture around it. Coagulation testing done subsequent to this event was found to be normal.

We have not encountered such a problem before and have not read of it in the literature. We wonder if anyone has been in a similar situation and what others would do. It is not uncommon to see some bleeding at the site of an epidural, but the bleeding is self-limited. This may warrant frequent inspection of the epidural insertion site.

\author{
Chidambaram Ananthanarayan MD FRCPC \\ Susan Haley MD FRCPC \\ Department of Anaesthesia \\ Mount Sinai Hospital \\ 600 University Avenue \\ Toronto, Ontario \\ M5G 1 X5
}

\section{Epidural blood patch in treatment of chronic headache}

\section{To the Editor:}

We read with interest the article by Parris' regarding the use of epidural blood patch in treating chronic headache which resembled post-dural puncture headache. We recently reported a case of spontaneous low-CSF pressure headache treated successfully with an epidural blood patch $^{2}$ and would like to add our experience in reviewing this condition.

The spontaneous onset of postural headache was furst described by Schaltenbrand in $1938 .{ }^{3}$ He termed the syndrome "spontaneous aliquorrhoea," and proposed three possible mechanisms to explain its occurrence: (1) diminished CSF production, (2) increased CSF absorption and (3) CSF leakage.

Subsequent case reports suggest that leakage is the most likely cause. Nosik ${ }^{4}$ in 1955 was able to demonstrate leakage through a tear in the dural sleeve of a lumbar nerve root during myelography. Labadie in $1976,{ }^{5}$ using lumbar isotope cisternography was unable to demonstrate an actual site of CSF leakage; however, the rapid disappearance of dye from the subarachnoid space and the rapid appearance of isotope in the bladder suggests that CSF leakage into the epidural space was occurring. Baker in $1983^{\circ}$ reported two cases, and one of these was successfully treated with an epidural blood patch. This was the only previous case of spontancous low CSF pressure headache to our knowledge to have been treated with an epidural blood patch. 